\title{
OPTICALLY ACTIVE CENTERS IN Er-IMPLANTED SILICON*
}

\section{H. Przybylińska}

Institute of Physics, Polish Academy of Sciences

Al. Lotników 32/46, 02-668 Warszawa, Poland

W. Jantsch, G. Hendorfer, L. Palmetshofer

Institut für Experimentalphysik, Johannes Kepler Universität, 4040 Linz-Auhof, Austria

\section{R.J. Wilson and B.J. Sealy}

Department of Electronic and Electrical Engineering, University of Surrey Guildford, Surrey GU2 5XH, UK

We show that of all the optically active Er centers in silicon found after ion implantation and optimum annealing $\left(900^{\circ} \mathrm{C}\right)$ the isolated cubic interstitial $\mathrm{Er}$ is the dominant $\mathrm{PL}$ center above $100 \mathrm{~K}$. At lower anneal temperatures $\left(\approx 600^{\circ} \mathrm{C}\right)$ with later rapid thermal anneal at $900^{\circ} \mathrm{C}$ oxygen related centers also emit.

PACS numbers: $78.55 . \mathrm{Hx}$

The particular attraction of Er doped systems is that the transition between the lowest spin orbit levels of $\mathrm{Er}^{3+},{ }^{4} I_{13 / 2} \rightarrow{ }^{4} I_{15 / 2}$, occurs at $1.54 \mu \mathrm{m}$, which wavelength happens to coincide with the minimum loss region of silica-based optical fibers [1]. Due to the screening of the $4 f$ shell by outer shells the intra- $4 f$-shell emission depends little on the host and the line positions are practically temperature independent. One of the most intensely explored systems is Er-doped Si [1-7] as the advanced integration technology of silicon offers great application possibilities, not only for a temperature stable infrared light source, but also for on-chip and inter-chip optical data transfer.

The low solubility of erbium in $\mathrm{Si}$ requires the application of nonequilibrium doping techniques, from which implantation occurs to be the most promising. A large effort has been undertaken to define the optimum processing parameters, which would ensure both high Er incorporation and the removal of radiation defects by suitable annealing. Coimplantation with light ions leads to a substantial photoluminescence (PL) intensity increase [2] and coimplantation with oxygen, in particular, to higher Er concentrations without the formation of precipitates [3] and less efficient temperature quenching of the PL intensity [4]. Both

*Work supported by Fonds zur Förderung der Wissenschaftlichen Forschung Austria, and partially by the State Committee for Scientific Research (Republic of Poland) under grant No 8 S50102905. 
effects were believed to be related to the tendency of erbium to form complexes with codopants and held responsible for the much higher PL yield of Er implanted Czochralski-grown (CZ) material, which abounds in oxygen, as compared to the high-purity float zone (FZ) Si.

Recently we have resolved the energy structure of two types of optically active Er centers by high resolution optical spectroscopy [6]: (i) cubic symmetry centers which we relate, as proposed in Ref. [6], to isolated Er ions occupying interstitial lattice sites - those centers dominate the emission in well annealed FZ-Si samples implanted with low Er fluences; (ii) Er-oxygen complexes with a close to axial site symmetry (labeled further on in text as $\mathrm{O} 1$ and $\mathrm{O} 2$ centers), which dominate the PL in CZ-Si at low Er concentrations only. In addition we found a number of centers with low site symmetry giving rise to PL lines close to the main cubic transition at $6504.8 \mathrm{~cm}^{-1}$. We relate those centers to $\mathrm{Er}$ complexes with residual radiation defects as they dominate the PL for increased Er, or codopant doses. Those findings have questioned the validity of the usual assumptions concerning the nature of the optically active Er centers in silicon and the role played by oxygen in promoting the erbium emission. In this paper we
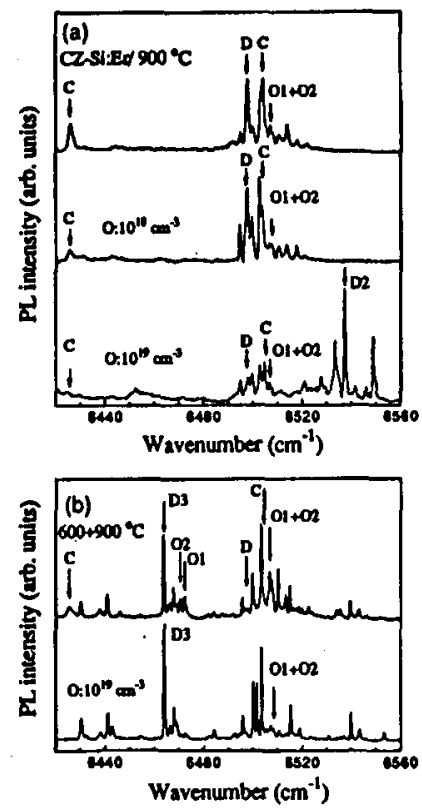

Fig. 1. PL spectra of CZ-Si implanted with $\mathrm{Er}$ at $2 \mathrm{MeV}$ to the peak concentration of $1.2 \times 10^{18} \mathrm{~cm}^{-3}$ and subsequently annealed at $900^{\circ} \mathrm{C}$ for $30 \mathrm{~min}$ (a) or $600^{\circ} \mathrm{C}$ for $30 \mathrm{~min}$ and $900^{\circ} \mathrm{C}$ for $15 \mathrm{~s}(\mathrm{~b})$, measured at $4.2 \mathrm{~K}$ with a resolution of $0.5 \mathrm{~cm}^{-1}$. The samples have been additionally coimplanted with oxygen to concentrations indicated in the figure. The lines labeled $\mathrm{C}$ belong to isolated, cubic interstitial $\mathrm{Er}$, those labeled $\mathrm{O} 1$ and $\mathrm{O} 2$ belong to axial symmetry Er-O complexes, whereas the one labeled $\mathrm{D}$ belongs to an Er-defect center. 
report on a study of the emission efficiency of the particular Er centers resolved by us earlier. We find that for deep Er implants ( $2 \mathrm{MeV}$ ) in CZ-Si the low temperature $\mathrm{PL}$ in well annealed samples $\left(900^{\circ} \mathrm{C}\right.$ for $\left.30 \mathrm{~min}\right)$ is dominated by the emission of isolated, interstitial Er centers as shown in Fig. 1 (the cubic center PL lines are labeled C) as well as Er-defect related centers (one of them is labeled D). Coimplantation with oxygen to a peak concentration corresponding to the Er peak concentrations leads only to an increase in the intensity of the low symmetry defect-related centers, with a substantial reduction of the cubic PL intensity. For an order of magnitude higher O-coimplant doses the cubic spectrum completely disappears and the simple Er-defect related lines become much weaker. Instead, quite different, broad and asymmetric PL lines appear (labeled D2) which might originate from extended Er-defect centers. A 30 min annealing at $600^{\circ} \mathrm{C}$ with additional rapid thermal anneal at $900^{\circ} \mathrm{C}$ for $15 \mathrm{~s}$, which is supposed to ensure a better recrystallization of heavily implanted samples, is shown to produce additional point-defect related centers (the strongest emission line is labeled D3). In all the investigated samples weaker PL lines of the axial, oxygen-related centers (O1 and $\mathrm{O} 2)$ can be observed. However, these centers are created already during Er implantation and subsequent sample processing influences their formation to a negligible extent.

Though the integrated PL intensity apparently increases with oxygen coimplantation, this is true only at low temperatures. At temperatures above $100 \mathrm{~K}$ only the emission of cubic and oxygen-related $\mathrm{O} 1$ and $\mathrm{O} 2$ centers remains. Careful investigations reveal that each center has a different temperature dependence even when measured in the same sample. At low temperatures the PL intensity is found to decrease with a deactivation energy of about $15 \mathrm{meV}$ for the Er-defect center

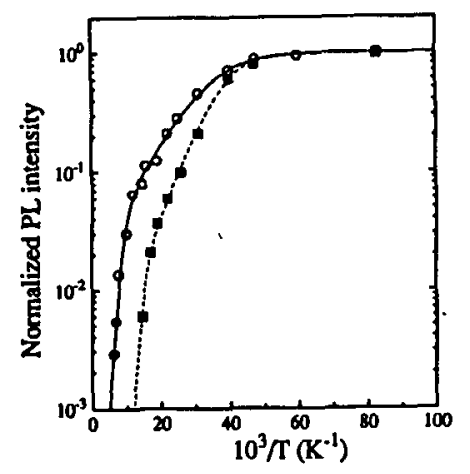

Fig. 2. Temperature dependence of the PL intensity for the interstitial cubic Er center (open circles) and the Er-D center (squares) as measured in a $p$-type sample implanted with $3 \times 10^{13} \mathrm{~cm}^{-2} \mathrm{Er}$ at $2 \mathrm{MeV}$. The drawn lines are fitted with the formula $I=$ $\left[1+C_{1} \exp \left(-E_{1} / k T\right)+C_{2} \exp \left(-E_{2} / k T\right)\right]^{-1}$ for the following parameters: $E_{1}=10 \mathrm{meV}$, $E_{2}=80 \mathrm{meV}, C_{1}=55, C_{2}=6.0 \times 10^{4}$ for the cubic center and $E_{1}=15 \mathrm{meV}$, $E_{2}=80 \mathrm{meV}, C_{1}=740, C_{2}=6.5 \times 10^{7}$ for the Er-D center. 
labeled D, $10 \mathrm{meV}$ for the $\mathrm{C}$ and $\mathrm{O} 1$ centers (the PL intensities of the so-called hot lines appearing for those centers with increasing temperature have been taken into account) and about $5 \mathrm{meV}$ for the line at $6504.1 \mathrm{~cm}^{-1}$, which is very close to the main cubic line but is defect related. The PL deactivation energies turn out to be a characteristic feature of a particular Er center and do not depend on the conduction type of the material, nor additional codoping. The values are typical of impurity bound exciton (BE) binding energies in Si. It seems that the erbium emission is excited via defect Auger recombination of excitons bound on the particular Er centers. For the low symmetry centers the trapping levels are most probably provided by the adjacent radiation defects. In fact, several energy levels have been observed in a deep level transient spectroscopy (DLTS) study of the investigated samples. The existence of a trap level related to an interstitial $\mathrm{Er}^{3+}$ impurity in Si has been predicted theoretically [7].

The thermalization of Er-BE is not, however, the main mechanism responsible for the temperature quenching of the Er PL intensity. As shown in Fig. 2 at elevated temperatures another de-excitation process appears with a deactivation energy of $80 \mathrm{meV}$ both for the $\mathrm{C}$ and $\mathrm{D}$-centers but with the efficiency depending strongly on the kind of emitting Er center. So far it is not clear whether the quenching is related to Auger type, nonradiative recombination processes within Er.

\section{References}

[1] H. Ennen, J. Schneider, G. Pomrenke, A. Axmann, Appl. Phys. Lett. 43, 943 (1983).

[2] J. Michel, J.L.Benton, R.F. Ferrante, D.C. Jacobson, D.J. Eaglesham, E.A. Fitzgerald, Y.H. Xie, J.M. Poate, L.C. Kimerling, J. Appl. Phys. 70, 2672 (1991).

[3] F. Priolo, S. Coffa, G. Franzo, C. Spinella, A. Carnera, V. Bellani, J. Appl. Phys. 74, 4936 (1993).

[4] S. Coffa, G. Franzo, F. Priolo, A. Polman, R. Serna, Phys. Rev. B 49, 16313 (1994).

[5] F.Y.G. Ren, J. Michel, Q. Sun-Paduano, B. Zheng, H. Kitagawa, D.C. Jacobson, J.M. Poate, L.C. Kimerling, Mater. Res. Soc. Symp. Proc. 301, 87 (1993).

[6] H. Przybylińska, G. Hendorfer, M. Bruckner, L. Palmetshofer, W. Jantsch, Appl. Phys. Lett. 66, 490 (1995).

[7] M. Needels, M. Schlüter, M. Lannoo, Phys. Rev. B 47, 15533 (1993). 